\title{
Implementación de la política de primera infancia en Cartagena, Colombia
}

\author{
Rosaura Arrieta-Flórez, Ph. D. ${ }^{a}$ \\ Universidad de Cartagena, Colombia \\ Lina Luz Córdoba-Girado. ${ }^{b}$ \\ Fundación Universitaria Tecnológico Comfenalco, Colombia \\ Rafaela Sayas-Contreras, Ph. D. ${ }^{\circ}$ \\ Universidad de Cartagena, Colombia
}

ipreg2@unicartagena.edu.co

\section{Resumen (analítico) \\ El interés superior de los niños y las niñas es obligación del Estado colombiano, propuesto por la Carta Política e implementado a través de políticas públicas. Sin embargo, las condiciones materiales no reflejan la prevalencia de la protección integral preconizada por las normas. Este estudio combina el análisis cualitativo y cuantitativo, e indaga las dinámicas de la implementación de la política pública de primera infancia en Cartagena (Bolívar), con captura de información primaria de funcionarios de diferentes niveles de gobierno, agentes comunitarios y organizaciones sociales. Los hallazgos muestran ba- rreras en la implementación de la política en materia de gestión ad- ministrativa, articulación institucional, atención educativa e inversión pública, principales causantes del acceso desigual a los derechos de primera infancia en la ciudad.}

\section{Palabras clave}

Bienestar de la infancia, política social, primera infancia, derechos del niño, cuidado del niño, educación de la primera infancia.

\section{Thesauro}

Tesauro de Ciencias Sociales de la Unesco.

\section{Para citar este artículo}

Arrieta-Flórez, R., Córdoba-Giraldo, L. L., \& SayasContreras, R. (2021). Implementación de la política de primera infancia en Cartagena, Colombia. Revista Latinoamericana de Ciencias Sociales, Niñez y Juventud, 19(3), 1-24.

https://dx.doi.org/10.11600/rlcsnj.19.3.4943

\section{Historial}

Recibido: 21.08 .2020

Aceptado: 27.12 .2020

Publicado: 20.08.2021

\section{Información artículo}

El artículo hace parte de una serie de trabajos realizados en el marco de la línea de investigación Políticas Públicas y Derechos Humanos del Instituto de Políticas Públicas de la Universidad de Cartagena; y de una investigación más amplia sobre la política pública de primera infancia en Colombia y su impacto en la generación de capacidades en niños y niñas menores de cinco años, realizada por Rosaura Arrieta-Flórez entre febrero de 2015 y febrero de 2019. Este proyecto no recibió financiación pública ni privada. Área: ciencias políticas. Subárea: administración pública. 


\section{Implementation of an Early Childhood Policy in Cartagena, Colombia}

\section{Abstract (analytical)}

The best interests of the child is an obligation of the Colombian State, proposed in its Constitution and implemented through public policies. However, the material conditions for children in the country do not reflect the prevalence of the comprehensive protection recommended in these standards. This study combines qualitative and quantitative analysis to investigate the dynamics of the implementation of the Early Childhood public policy in Cartagena (Bolívar). The research collected primary information from officials at different levels of government, community agents and social organizations. The findings highlight barriers for the implementation of this policy in the areas of administrative management, institutional coordination, educational services and public investment, concluding that these are the main causes of unequal access to early childhood rights in the city.

Keywords

Child welfare, social policy, early childhood, children's rights, child care, early childhood education.

\section{Implementação da Política da Primeira Infância em Cartagena, Colombia}

\section{Resumo (analítico)}

O maior interesse para as crianças é a obrigação do Estado colombiano, proposta na Carta Política e implementada por meio de políticas públicas. No entanto, as condições materiais não refletem a prevalência da proteção abrangente recomendada pelas normas. Este estudo combina análises qualitativas e quantitativas, e investiga a dinâmica de implementação da política pública da Primeira Infância em Cartagena (Bolívar), com a captação de informações primárias de funcionários de diferentes níveis de governo, agentes comunitários e organizações sociais. Os achados mostram barreiras na implementação da política em questões de gestão administrativa, coordenação institucional, atenção educacional e investimento público, principais causas da desigualdade de acesso aos direitos da Primeira Infância no município.

\section{Palavras-chave}

Bem-Estar Infantil, Política Social, Primeira Infância, Direitos da Criança, Assistência à Criança, Educação Infantil.

\section{Información autoras}

[a] Profesora Investigadora Instituto de Políticas Públicas, Universidad de Cartagena, Colombia. Candidata a Doctora en Ciencias Sociales, Universidad Pablo de Olavide. iD 0000-0002-2026-6899. H5: 0. Correo electrónico: ipreg2@unicartagena.edu.co

[b] Profesora Investigadora, Fundación Universitaria Tecnológico Comfenalco, Colombia. Trabajadora Social, Magíster en Derechos Humanos, Interculturalidad y Desarrollo, Universidad Internacional de Andalucía. H5: 0. Correo electrónico: linacordoba7@gmail.com iD 0000-0001-5550-4124.

[c] Profesora investigadora de la Universidad de Cartagena, Colombia. Doctora en Sociología, Universidad de Belgrano (Argentina). iD 0000-0001-9342-3907. H5: 0. Correo electrónico: rsayasc@unicartagena.edu.co 


\section{Introducción}

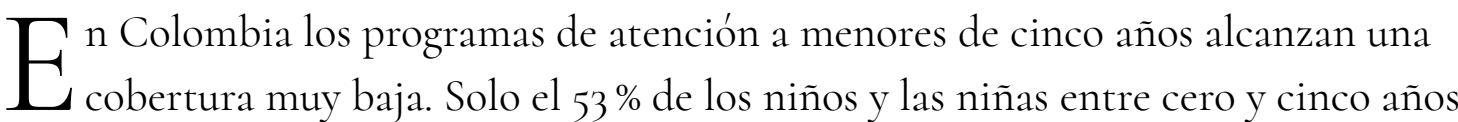
acceden a alguno de los programas estatales (Bernal, 2014), mientras que los indicadores de salud y acceso a servicios de esta población evidencian inequidades sociales, así como la necesidad de fortalecer y, en algunos casos, reorientar la política pública (Arrieta-Flórez \& Caro-Gómez, 2010)

Los resultados en salud también confirman la presencia de inequidades: según la Encuesta Nacional de Demografía y Salud, el $26 \%$ de los niños menores de 5 años - del quintil de riqueza más bajo- sufren de anemia nutricional, en comparación con el 19\% del quintil más alto, a pesar de la cuantiosa financiación pública de los últimos años (en 2018 superó los USD 1481 millones). Esta brecha se agrava en las zonas rurales y en la llamada periferia colombiana, donde se encuentran gran parte de los hogares afrodescendientes e indígenas, creando un círculo vicioso que perpetúa la inequidad y reproduce las trampas de la pobreza (Rodríguez \& Benavides 2016).

En Cartagena, donde se registra la mayor tasa de exclusión entre las principales ciudades colombianas (Ayala \& Meisel, 2016), se evidencia una deuda con la garantía de los derechos básicos de los menores de cinco años. Las tasas de mortalidad por enfermedades prevenibles, como las respiratorias agudas, son casi dos veces la de Colombia (22 muertes por cada 100 mil habitantes) y la mortalidad por desnutrición crónica es del 7.33\%.

Los indicadores de cuidado infantil también son preocupantes en la ciudad: según el programa Cartagena Cómo Vamos, en dicha ciudad viven alrededor de 100 mil niños y niñas menores de cinco años: el $41 \%$ permanece en casa con un adulto y el $11 \%$ queda al cuidado en casa de otros menores o fuera de la casa con amigos o familiares; es decir, no acceden a los programas para el cuidado y la atención integral a la primera infancia.

Esta investigación tiene como objetivo describir y analizar la implementación de la política pública de primera infancia en Cartagena de Indias, con el fin de identificar las 
principales barreras y oportunidades para la garantía de los derechos de los niños y las niñas menores de cinco años de la ciudad, aportando además insumos que permitan el logro de su objetivo superior: la protección integral de la primera infancia.

Partimos de la hipótesis de que, a pesar de la existencia de una política pública que prioriza la atención y la inversión en la primera infancia, la oferta de servicios en Cartagena es desigual y desarticulada, mientras que los recursos para su funcionamiento a nivel territorial no están garantizados; por tanto, su impacto en la inclusión social de los más pobres y la reducción de inequidades es marginal.

Todo lo anterior sucede al mismo tiempo que se fortalecen y generalizan los argumentos científicos, éticos y los avances normativos alrededor de la importancia de la inversión en la primera infancia.

La Convención Internacional sobre los Derechos del Niño, aprobada por la Asamblea General de las Naciones Unidas en 1989, marcó un hito en la historia de la protección de los derechos de los niños y las niñas en el mundo. Según la Comisión Económica para América Latina y el Caribe y el Fondo de las Naciones Unidas para la Infancia (2018) es el «primer tratado vinculante en relación con el cumplimiento de los derechos civiles, políticos, sociales, económicos y culturales de la infancia» (p. 9). En la actualidad se estructura como el tratado de derechos humanos más ratificado a nivel internacional, lo cual es indicativo del consenso universal en torno a la protección y al desarrollo infantil (Gómez, 2007).

En muchos Estados firmantes como Colombia se logró la institucionalización de programas y políticas que potenciaran el desarrollo humano en los primeros años de vida y las inversiones públicas para el aseguramiento de la atención integral y de calidad (Black et al., 2017; Nores \& Barnett, 2010; ONU, 2005).

La observación n. ${ }^{\circ} 7$ de las Naciones Unidas señala que la primera infancia es una etapa esencial para la realización de los derechos de la infancia, constituyéndose en «el período de más rápido crecimiento y cambio de todo su ciclo vital, en términos de maduración del cuerpo y sistema nervioso, de movilidad creciente, de capacidad de comunicación y aptitudes intelectuales, y de rápidos cambios de intereses y aptitudes» (§ 6). Bajo estos presupuestos, se insta a los Estados a adoptar planes estratégicos para el desarrollo de la primera infancia, desde un marco de derechos y, por consiguiente, a aumentar la asignación de recursos humanos y financieros a los servicios y programas para este grupo poblacional (Fondo de las Naciones Unidas para la Infancia, 2006). 
En Colombia, con la Ley 1098 - Ley de infancia y adolescencia - (Congreso de la República de Colombia, 2006) se definió el compromiso de los gobiernos territoriales de formular e implementar políticas públicas para dar cumplimento a las obligaciones del Estado frente a los derechos de la infancia y la adolescencia. Si bien dentro de esta política se incluyó a la primera infancia como una etapa dentro del ciclo de vida, solo hasta 2016 (con la Ley 1804; Congreso de la República de Colombia, 2016) el Estado colombiano establece la Política pública para el desarrollo integral de la primera infancia (De cero a siempre) para los niños y las niñas menores de cinco años, como política de obligatorio cumplimiento en todo el territorio colombiano. Esta busca asegurar el desarrollo integral y la garantía del goce efectivo de los derechos de los menores de cinco años a través de acciones que materialicen las condiciones requeridas en salud, nutrición, cuidado y educación inicial.

A pesar de la relevancia del tema y de la importancia en la agenda pública mundial y nacional, la literatura especializada registra pocas investigaciones sobre el alcance, pertinencia e impacto de esta política pública. A nivel internacional existe consenso sobre los beneficios individuales y sociales de los programas de cuidado y atención a la primera infancia (Baker et al., 2019; Sheldom, 2019), pero pocas evaluaciones de los impactos de las políticas. Nores y Barnett (2010) encontraron, mediante un estudio cuasiexperimental de 38 intervenciones en 23 países en Europa, Asia, África, Centro y Sur América, que los programas que combinan intervenciones educativas y de cuidado tienen mayores efectos cognitivos si se comparan con los programas de transferencias condicionadas de dinero o intervenciones únicamente nutricionales. Por su parte, Leseman y Slot (2020) encontraron que existe evidencia sólida de que los programas de alta calidad para la primera infancia, dirigidos a grupos desfavorecidos, pueden tener beneficios duraderos y un alto rendimiento de la inversión.

En Colombia sobresalen la serie de trabajos realizados por Bernal et al. (2009), Bernal y Camacho (2010) y Bernal (2014, 2019), quienes caracterizan y evalúan el impacto de los programas de atención inicial ofertados por el Instituto Colombiano de Bienestar Familiar (ICBF). Los resultados reportan diferentes barreras de acceso con calidad de la primera infancia a los programas de atención inicial en Colombia que, según Bernal et al. (2009), Bernal y Camacho (2010, 2012), se reflejan en un alto número de niños y niñas sin acceso a atención o cuidado de mala calidad, una brecha entre zonas rurales y urbanas y la deficiente supervisión a los procesos desarrollados por el personal docente. Adicionalmente, se identifican falencias en el seguimiento y evaluación de la política pública 
(Bernal, 2014), una inapropiada infraestructura institucional y debilidades en la consolidación del trabajo intersectorial (Bernal \& Ramírez, 2019).

Este trabajo aporta a la literatura existente, en la medida que se constituye en el primer estudio que analiza en profundidad el caso de Cartagena de Indias, reivindica las voces de los diferentes actores de la política pública en la ciudad y combina técnicas propias de la investigación cuantitativa y cualitativa para un análisis más integral. En este sentido, contribuye con evidencia científica a la comprensión de las causas políticas, institucionales y sociales que limitan el acceso a la atención con calidad, igualdad y pertinencia de los niños y las niñas menores de 5 años, proponiendo líneas de acción para la transformación positiva de estos.

\section{Metodología}

La investigación se desarrolló a través del método mixto y se soportó en un diseño de integración de procesos (Hernández-Sampieri \& Mendoza, 2018) que permitió combinar de forma simultánea la recolección y el análisis de datos cualitativos y cuantitativos durante todo el proceso de estudio. El propósito fue realizar inferencias producto de toda la información recabada (metainferencias) y lograr un mayor entendimiento del fenómeno bajo estudio. Se asignó mayor prioridad a los datos cualitativos y, mediante la integración con los datos cuantitativos, se buscó triangular, complementar y validar la información, descubriendo nuevas variables y categorías de análisis (Hernández-Sampieri \& Batista 2014).

La investigación se direccionó en identificar, a partir de las categorías de gestión administrativa, articulación institucional, atención educativa e inversión pública, las principales limitaciones en la implementación de la política pública de infancia (en adelante PPI) en Cartagena, atendiendo a la existencia de un estudio previo realizado sobre la misma en el año 2015. Es decir, con el objetivo de dar continuidad al proceso investigativo se identificaron los avances y retrocesos de la PPI, esta vez articulando el análisis en función del cumplimiento de los principios de la Convención Internacional sobre los Derechos del Niño bajo un enfoque de derechos y diferencial.

La primera fase de la investigación (fase conceptual), utilizó como técnicas principales la revisión y el análisis documental de las líneas de acción propuestas por la Comisión Intersectorial para la Atención Integral a la Primera Infancia (CIPI) y los lineamientos 
técnico-operativos diseñados para la operación de los programas de primera infancia en el país, esto con el fin de identificar los procesos intersectoriales requeridos para la ejecución de la PPI a nivel territorial.

En la fase empírica metodológica (recolección de información) se identificaron a los actores claves en el territorio y se desarrollaron acercamientos institucionales (ICBF y Alcaldía) para articular acciones; de ello, la selección de zo centros de atención y cuidado bajo los criterios de ubicación geográfica, cobertura de la atención y consentimiento informado para la participación. Además, se realizaron entrevistas estructuradas a funcionarios del ICBF Sección Asistencia Técnica (en adelante, ICBF-SAT) y de la Alcaldía Mayor de Cartagena de Indias, a coordinadores administrativos, agentes pedagógicos/ madres comunitarias de los centros y actores del tercer sector (tabla 1 ).

\section{Tabla 1}

\section{Población participante, 2015-2019}

\begin{tabular}{lcccc}
\hline \multirow{2}{*}{ ENTREVISTAS } & & \multicolumn{2}{c}{. $^{\circ}$ de entrevistas } \\
\cline { 5 - 5 } & $\mathbf{2 0 1 5}$ & & $\mathbf{2 0 1 9}$ \\
\hline AlcBF Regional Bolívar & & & & 4 \\
Coordinadores Administrativos CDI/HI & & 20 & & 20 \\
Agentes docentes/madres comunitarias & & 25 & & 27 \\
Actores tercer sector & & & & 2 \\
Total participantes & & $\mathbf{5 4}$ & & $\mathbf{5 8}$ \\
Total unidades participantes & & $\mathbf{1 9}$ & & $\mathbf{2 0}$ \\
\hline
\end{tabular}

En la fase empírica analítica (análisis de resultados), para el estudio de las categorías gestión administrativa y articulación institucional, se usó el software Atlas.ti 5.o, que permitió sistematizar e interpretar los datos aportados por las entrevistas. En la categoría de atención educativa se utilizó la técnica representación gráfica para la descripción y explicación de los datos de atención por modalidad reportados por el ICBF para los dos años de estudio, análisis triangulado con los resultados de las entrevistas y la observación participante.

En la categoría inversión pública se utilizó el software Stata 13.0 para tramitar los datos reportados en el Formulario Único de Registro del Departamento Nacional de Planeación (DNP) para Cartagena y otras 6 ciudades colombianas, y se construyeron indicadores que dan cuenta de la magnitud de la inversión en primera infancia, la fuente y el 
uso de los recursos de inversión. Este ejercicio se complementó con la revisión de la política pública distrital e información recabada en las entrevistas. La tabla 2 ilustra la metodología desarrollada por categorías de análisis, técnicas y fuentes.

Tabla 2

Resumen metodológico

\begin{tabular}{|c|c|c|c|}
\hline Categoría & Temas/subcategorías & Técnicas/tipo de análisis & Fuentes \\
\hline Gestión & \multirow{2}{*}{$\begin{array}{l}\text {-Procesos administrativos } \\
\text {-Operación de los programas } \\
\text {-Gestión intersectorial } \\
\text {-Garantía institucional de } \\
\text { derechos. } \\
\text {-Tránsito armónico }\end{array}$} & \multirow[b]{2}{*}{$\begin{array}{l}\text {-Revisión y análisis documental } \\
\text {-Entrevistas estructuradas } \\
\text {-Análisis de datos cualitativos }\end{array}$} & $\begin{array}{l}\text { ICBF-SAT } \\
\text { FAS }\end{array}$ \\
\hline $\begin{array}{l}\text { Articulación } \\
\text { institucional }\end{array}$ & & & $\begin{array}{l}\text { Agentes pedagógicas } \\
\text { Línea base } 2015 \\
\text { Linimentos operativos } \\
\text { ICBF-CIPI-MEN. } \\
\text { Tercer sector }\end{array}$ \\
\hline Atención educativa & $\begin{array}{l}\text {-Oferta institucional } \\
\text {-Atención por modalidad de }\end{array}$ & -Revisión y análisis documental & $\begin{array}{l}\text { ICBF } \\
\text { Línea Base } 2015\end{array}$ \\
\hline Inversión pública & $\begin{array}{l}\text { - atención diferencial } \\
\text {-Infraestructura institucional } \\
\text {-Inversión en PI. } \\
\text {-Tendencias de la inversión } \\
\text {-Fuente recursos de inversión } \\
\text {-Usos recursos de inversión }\end{array}$ & $\begin{array}{l}\text {-Entrevistas estructuradas } \\
\text {-Cartografía institucional } \\
\text {-Análisis de datos cualitativos } \\
\text { y cuantitativos } \\
\text {-Representación gráfica }\end{array}$ & $\begin{array}{l}\text { Linimentos Operativos } \\
\text { ICBF-Cipi-MEN } \\
\text { Agentes Participantes } \\
\text { Tercer Sector } \\
\text { FUT-DNP }\end{array}$ \\
\hline
\end{tabular}

\section{Resultados}

Esta sección analiza los hallazgos de los datos recopilados y procesados de 112 entrevistas realizadas entre los años 2015 y 2019, y presenta los resultados agrupados en cuatro categorías de análisis: gestión administrativa, articulación institucional, atención educativa e inversión pública.

\section{Gestión administrativa e institucional para el desarrollo de la política pública}

La gestión administrativa para la ejecución de la PPI en los territorios colombianos recae, principalmente, en el ICBF y en las entidades territoriales. En el caso de Cartagena de Indias, el ICBF se encarga de contratar a operadores que fungen como entidades administradoras del servicio (en adelante EAS), las cuales, a partir de sus obligaciones contractuales, deben coordinar a los profesionales que intervienen en las diferentes modalidades de atención. 
De igual forma, el ICBF desembolsa recursos representados en una canasta de atención y aporta la línea técnica a las EAS para la operación de las modalidades institucionales (Centros de Desarrollo Infantil —en adelante CDI- y Hogares Infantiles), familiares (Hogares Fami y desarrollo en medio familiar) y comunitarias (Hogares Comunitarios de Bienestar —en adelante $\mathrm{HCB}-$ ).

La contratación se realiza en el marco del Banco Nacional de Oferentes de la Dirección de Primera Infancia, bajo algunos criterios como capacidad operativa, índice de desempeño de la entidad y de la unidad aplicativa, ${ }^{1}$ información que se obtiene a través de las visitas que el ICBF realiza a las unidades (ICBF-SAT, 2019).

Una vez adjudicado el contrato, las EAS deberán: conformar el equipo de trabajo, focalizar y caracterizar a la población; formular el Plan operativo de atención integral; garantizar, en conjunto con las entidades del SNBF, la protección de los derechos de los niños y las niñas participantes; articular sus acciones con las familias, la comunidad y organizaciones sociales presentes en el territorio; y realizar seguimiento individualizado al proceso de los niños y las niñas (ICBF-SAT, 2016). La figura 1 sintetiza este proceso.

\section{Figura 1}

\section{Ruta administrativa para la operación de modalidades}

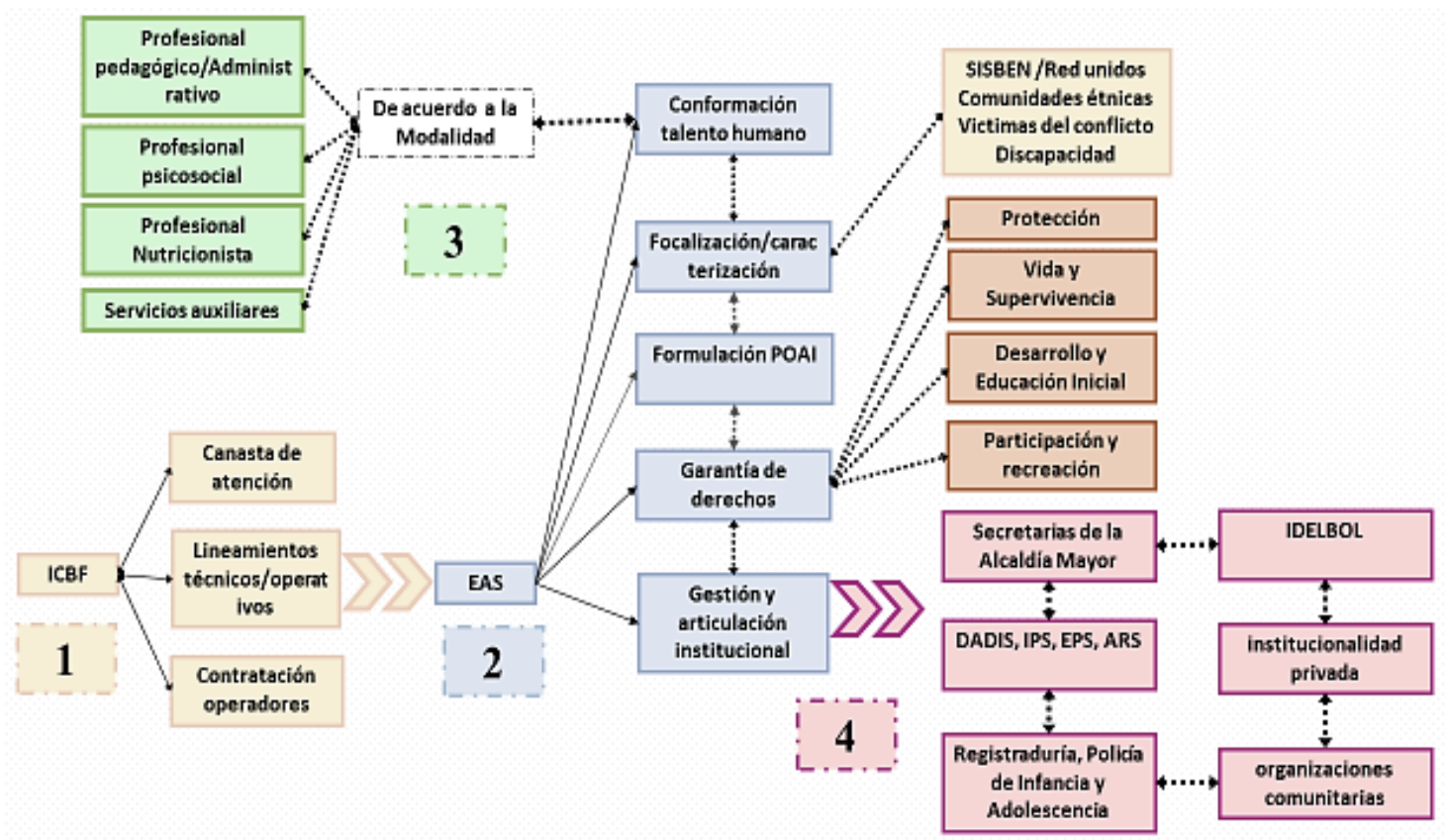

Nota. Basado en entrevistas estructuradas y lineamentos operativos ICBF (2020).

\footnotetext{
${ }^{1}$ Corresponde al lugar físico donde se presta el servicio a los usuarios de cada modalidad de primera infancia.
} 
De acuerdo con los entrevistados, lo procedimientos administrativos descritos anteriormente no han sido modificados; no obstante, en el año 2019 el proceso de contratación presentó cambios en los criterios de selección de las EAS, lo que generó inconformidades sociales denunciadas en los medios de comunicación local por parte de las EAS y las familias. De igual forma, las EAS denunciaron que, aun cumpliendo con los requisitos solicitados en el Banco de Oferentes, los contratos no fueron renovados o adjudicados y tampoco recibieron notificación formal del ICBF sobre los criterios de selección de operadores.

Nosotros tenemos más de 16 años de tener un contrato de aporte con el Instituto Colombiano de Bienestar Familiar. Desde el año pasado que comenzó este proceso de contratación todo ha sido atípico, porque los que han establecidos este año son nuevos, tanto para ellos [ICBF] como para nosotros, que somos operadores. A pesar de haber cumplido con los requisitos, nos hemos quedado por fuera de la contratación y no tuvimos ninguna notificación formal del ICBF. Coordinadora Pedagógica (Chimá, 202ob)

Si bien los retrasos en la contratación son un denominador común en los dos períodos de estudio, en 2019 los entrevistados coinciden en que las decisiones administrativas han generado, además de demoras en el inicio de la atención, una gran inconformidad manifestada en movilizaciones por parte de los padres de familia, las madres Fami en tránsito, tutoras, docentes, agentes educativos y demás personas que trabajan con los operadores.

Después decidimos marchar hasta el Centro, hasta las instalaciones del ICBF, para poner en conocimiento nuestro descontento, puesto que ahora no sabemos a dónde tenemos que llevar a nuestros hijos, pues este es el CDI más cercano. Padre de Familia (Chimá, 2020a)

La inestabilidad en la contratación de las EAS afecta también las sinergias institucionales (articulación intersectorial) que deben generarse entre la Alcaldía Mayor y sus instituciones descentralizadas, con competencias en la atención integral de la primera infancia y los operadores. Para ello, las EAS, de acuerdo con las áreas del derecho de los niños y las niñas, identifican a la entidad territorial responsable de garantizarlo y, mediante cartas de articulación, le solicitan formalmente la protección del derecho; vale la pena resaltar que solo en situaciones de resistencia institucional interviene el ICBF (figura 1).

De acuerdo con la información recopilada en las entrevistas en los años 2015 y 2019, se evidencia una heterogeneidad en los procesos de articulación institucional. Por un lado, 
permanecen alianzas positivas entre las EAS y entidades como Secretaría de Educación, Salud, Dadis, también con las entidades promotoras de salud, los Centros Zonales ICBF y Migración Colombia, reflejadas en frecuentes jornadas de vacunación, odontología y desparasitación en las unidades aplicativas, el aseguramiento de los cupos en la educación preescolar y el acceso de los niños a escenarios de recreación. Sin embargo, se encuentra que otras entidades determinantes en la garantía de los derechos de los niños y las niñas en la ciudad no responden oportunamente a las solicitudes de las EAS.

La Registraduría no cumple. Por ejemplo, en Barú tenemos la necesidad del registro civil; entonces cuando la EAS le dice a la Registraduría que los papitos no pueden venir a la ciudad, por el costo del pasaje; por tanto, se les solicita que se trasladen a Barú; entonces la registradora no llega, y son 50 niños o zo niños que necesitan atención. (ICBF-SAT, 2019)

La desarticulación se evidencia también en las gestiones institucionales para asegurar el tránsito armonioso de los niños y las niñas a la educación formal y se refleja (según las entrevistas realizadas tanto en 2015 como en 2019) en la dificultad para que la Secretaría de Educación, el Ministerio de Educación Nacional y el ICBF construyan las condiciones pedagógicas necesarias para la continuidad en el preescolar de los procesos desarrollados por los programas de primera infancia.

Realmente hay un trauma y persiste el trauma; porque, a pesar de que hay una guía que orienta los servicios de educación inicial y la educación formal, hay una ruptura. Las docentes del preescolar se guían de unos estándares, un índice de desempeño que mide la educación formal, diciendo que el niño de transición debe saber leer, debe aprenderse los números del 1 al 10o, o sea, ¿cómo no va a existir esa ruptura si desde la educación inicial no se le enseña academia al niño, se le apunta al desarrollo infantil? Entonces, cuando el niño llega a transición empieza el comentario de la profesora que el ICBF no enseña nada, que los niños del CDI van a jugar, a comer y a dormir. (Coordinadora, CDI-18, 2019)

Los lineamientos pedagógicos del Ministerio de Educación Nacional y el ICBF difieren tanto en los planteamientos teóricos como en la práctica pedagógica; así, mientras la PPI promueve el aporte de experiencias significativas mediante el juego, la literatura, la exploración al medio y el arte (CIPI, 2013), la educación formal busca medir el desarrollo de competencias del ser, saber y hacer, bajo metodologías e índices idóneos para ello (Rodríguez, 2014). Los actores señalan que es necesario construir solidas sinergias institucionales entre el ICBF, el Ministerio de Educación y la Secretaría de Educación, que 
reconozcan y valoren las experiencias y aprendizajes potenciados mediante los programas de primera infancia en la ciudad.

Si el Ministerio de Educación no flexibiliza sus guías y sus estándares no se puede hacer nada. El trabajo de ICBF y Ministerio de Educación es nulo, porque si tú, como educador, no creas conciencia y dices «este niño viene de la educación inicial donde aprende esto», es decir, no se retoma del trabajo previo, no se puede hacer nada. (Agente pedagógica-10, 2019)

Un avance identificado en 2019, respecto al año 2015, es la creación de la Mesa de Tránsito Armónico en la ciudad. La mesa es un espacio de deliberación entre el Distrito, las EAS, el ICBF y la Secretaría de Educación, y tiene como fin establecer estrategias para la garantía del tránsito armonioso de la primera infancia al preescolar. Sin embargo, los participantes coinciden en que los compromisos pactados en este escenario «se quedan alli» (Coordinadora, CDI-13, 2019) y no se traducen en intervenciones concretas.

Nosotros manifestamos los problemas en la mesa, pero todo queda allí, no se hace nada; todavía es la hora que algunas profesoras de los colegios no quieren aceptar a los niños del ICBF porque, según ellas, no saben contar, ni leer. Hay municipios y departamentos que han avanzado en eso y el trabajo lo tienen bien avanzado, pero en Bolívar eso no está definido todavía. (ICBF-SAT, 2019)

Las madres comunitarias plantean que, a pesar de la importancia de la mesa como espacio de articulación y toma de decisiones, su papel se ha limitado al aseguramiento de los cupos educativos al preescolar de los niños y las niñas de la educación inicial.

Eso no es una mesa como tal. Las madres comunitarias con los actores de estos escenarios hacen un trabajo de articulación con la escuela regular para concertar la posibilidad de que los niños que ellas están atendiendo, cuando cumplan los 5 años puedan acceder a un cupo en esta institución educativa. El relacionamiento se da en términos de garantizar el acceso, pero no para que pedagógicamente el tránsito sea armonioso y exitoso. (Madre comunitaria-18, 2019)

En síntesis, la triangulación de la información aportada por agentes administrativos y pedagógicos de las EAS, los funcionarios del ICBF y de la Alcaldía Mayor permite afirmar que para mejorar la gestión administrativa de la PPI en la ciudad es necesario garantizar la transparencia y la oportunidad en la contratación; asimismo, fortalecer los espacios existentes para la articulación y seguimiento institucional a los procesos, de tal forma que 
se logre la integralidad en los procesos y la sostenibilidad de las acciones (Bernal \& Ramírez, 2019).

\section{Atención educativa e inversión pública en la política pública De cero a siempre}

En Cartagena, los programas de primera infancia atienden a madres gestantes y lactantes en las modalidades de desarrollo infantil en medio familiar y Hogares Fami; a niños y niñas de 2 a 5 años en CDI, hogares infantiles y hogares comunitarios de bienestar. Además, el componente étnico funciona mediante la atención propia e intercultural y se desarrolla en territorios étnicos, zonas rurales y rurales dispersas de la ciudad. De acuerdo con el análisis de los datos cuantitativos, entre 2015 y 2019 se observa un aumento promedio anual del $14 \%$ del total de niños y niñas atendidos en todas las modalidades. La modalidad con mayor incremento fue la de hogares comunitarios, que pasó de atender 4830 niños y niñas en 2015 a 18 oo5 en 2019, es decir, un aumento promedio anual del $54 \%$. No obstante, este aumento contrasta con la reducción promedio anual del $14 \%$ de la atención registrada en CDI (figura 2).

figura 2

Atención a la primera infancia Cartagena de Indias (2015-2019)

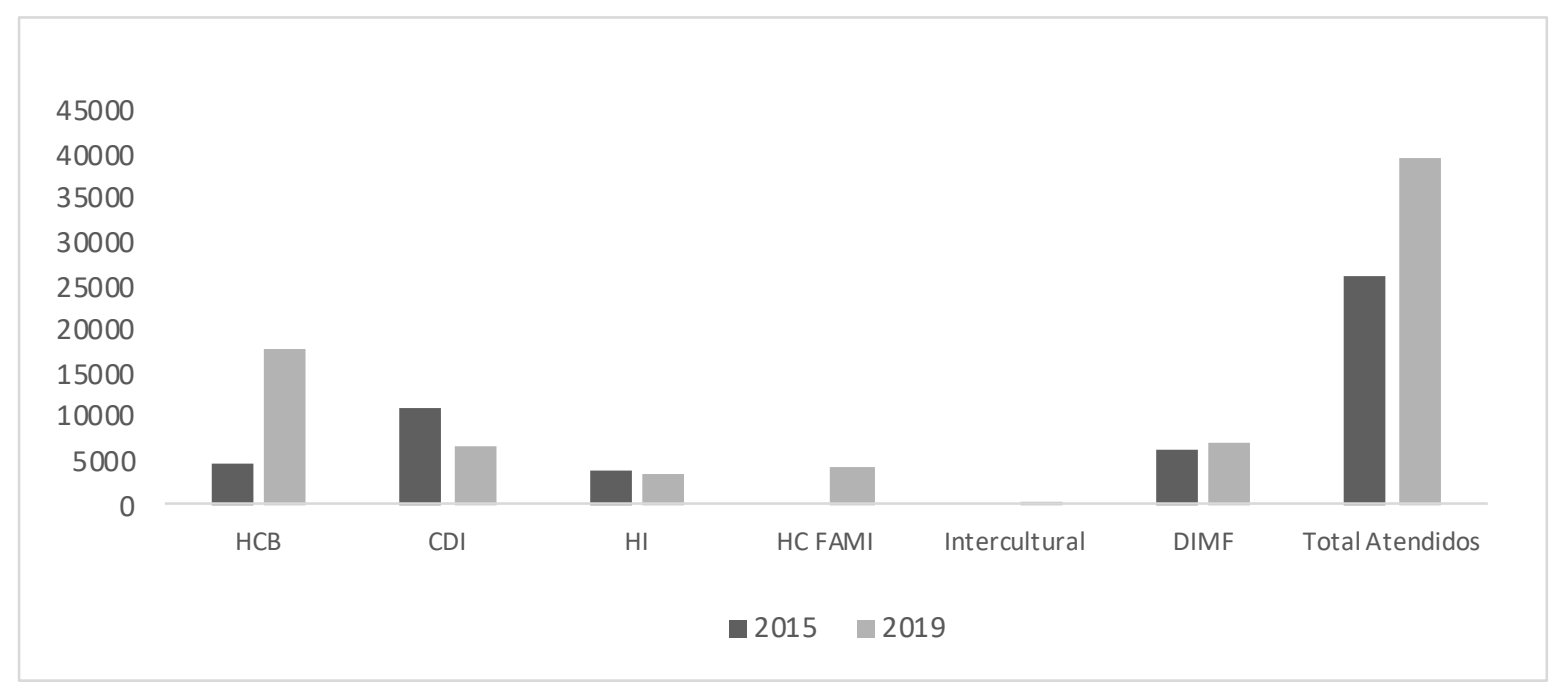

Nota. Basado en datos del ICBF (2019).

La reducción del número de niños y niñas atendidos en CDI puede considerarse un retroceso de la ciudad en su objetivo de aumentar la cobertura de atención integral. Y, 
entre otras razones, puede explicarse por la escasa infraestructura institucional existente en la ciudad. En 2019, del total de las unidades propias dispuestas para la atención y educación inicial, en Cartagena solo el 1.5\% corresponde a CDI y hogares infantiles, esto es, 50 unidades aplicativas. De ellas, 30 son institucionales (CDI y hogares infantiles) y zo familiares (Fami y desarrollo en medio familiar). Y, para el caso de los HCB, cada vivienda de la madre comunitaria corresponde a una unidad, es decir, funcionan 1855 (tabla 3 ).

Tabla 3

Número de unidades institucionales en Cartagena (2019)

\begin{tabular}{lcccc}
\hline \multicolumn{1}{c}{ Unidades del servicio } & CDI & hogares infantiles & Familiar & HCB \\
\hline Histórica y del Caribe Norte & 1 & 2 & 4 & 592 \\
Virgen y Turística & 14 & 6 & 10 & 754 \\
Industrial y de la Bahía & 2 & 5 & 6 & 509 \\
Total & 17 & 13 & 20 & 1855 \\
\hline
\end{tabular}

Nota. Basado en datos del ICBF (2019).

Para el año 2019, del total de niños atendidos en las diferentes modalidades en la ciudad, un poco menos de la mitad (el $45.6 \%$ ) es atendido en $\mathrm{HCB}$, seguido por la modalidad familiar (28.5\%) y los CDI, junto con los Hogares Infantiles, participan con el $24.4 \%$ (tabla 4). Esta tendencia es similar a la que presenta el país, en donde los HCB y HCB Fami concentran el $45 \%$ del total de la atención, mientras las modalidades integrales representan el $24.6 \%$, de los cuales solo el $11.7 \%$ corresponde a modalidades institucionales (ICBF, 2014).

\section{Tabla 4}

Atención por modalidad, Cartagena (2019)

\begin{tabular}{lc}
\hline Modalidad & Atención \\
\hline CDI (institucional) & 6621 \\
Hogares infantiles (institucional) & 3438 \\
HCB (comunitario) & 18005 \\
HCB-Fami & 4368 \\
Desarrollo infantil en medio familiar (familiar) & 6911 \\
Intercultural & 133 \\
Total general & $\mathbf{3 9 4 7 6}$ \\
\hline
\end{tabular}

Nota. Basado en datos del ICBF (2019). 
Los diferentes actores entrevistados señalaron que la limitada infraestructura física para la oferta de la atención institucional en los CDI y hogares infantiles es una limitación asociada a la inversión insuficiente de recursos y la desarticulación entre el ICBF y la Alcaldía Mayor de Cartagena, instituciones que tienen como función proponer y generar las acciones necesarias para garantizar la atención integral de la primera infancia más vulnerables de la ciudad; ello, en el marco de instancias de coordinación territorial, como la Mesa de Primera Infancia y el Consejo Distrital de Política Social.

Los problemas de infraestructura se informan a los consejos de política pública, a las mesas técnicas, pero siempre queda allí. (Coordinadora-15, 2019)

A nivel de infraestructura tenemos una dificultad muy grande, porque en Cartagena como tal, la infraestructura que ha dado el Distrito solo son la de Bicentenario, Flor del Campo y una que van a entregar en el Nelson Mandela. El responsable de infraestructura es la Secretaría de Infraestructura, es decir, los entes territoriales. Nosotros tenemos la responsabilidad de brindar la línea técnica, pero nosotros no construimos. (ICBF-SAT, 2019)

Señalan los participantes que el Distrito es responsable de garantizar la infraestructura para la atención, obligación emanada de las recomendaciones de las instancias de articulación y de la política Voces que hablan, voces que deciden, esta última desactualizada desde el año 2010. En dicha política, las metas para garantizar la educación inicial eran: 1) atender en el año 2015 al $100 \%$ de los niños y niñas menores de 6 años en medios institucionales y 2) ampliar la cobertura mediante la construcción de 3 jardines sociales y 3 hogares sociales (p. 12); objetivos que no se han cumplido.

Por otra parte, los datos de inversión pública en la primera infancia reflejan tres tendencias que explican las barreras político-administrativas para la garantía de los derechos de los niños y las niñas en Cartagena, entre ellas la existencia de infraestructura necesaria para la atención. En primer lugar, se evidencia un rezago de la inversión durante la última década; si bien entre 2010 y 2018 la inversión per cápita anual en primera infancia en la ciudad creció en promedio un $34 \%$, en términos monetarios esta inversión no superaba los 200 mil pesos en el año 2018 y representaba tan solo el $10 \%$ de lo invertido por ciudades como Barranquilla y Medellín en el mismo año (figura 3). 


\section{Figura 3}

Inversión per cápita en primera infancia en Cartagena y principales ciudades de Colombia (2010-2018)

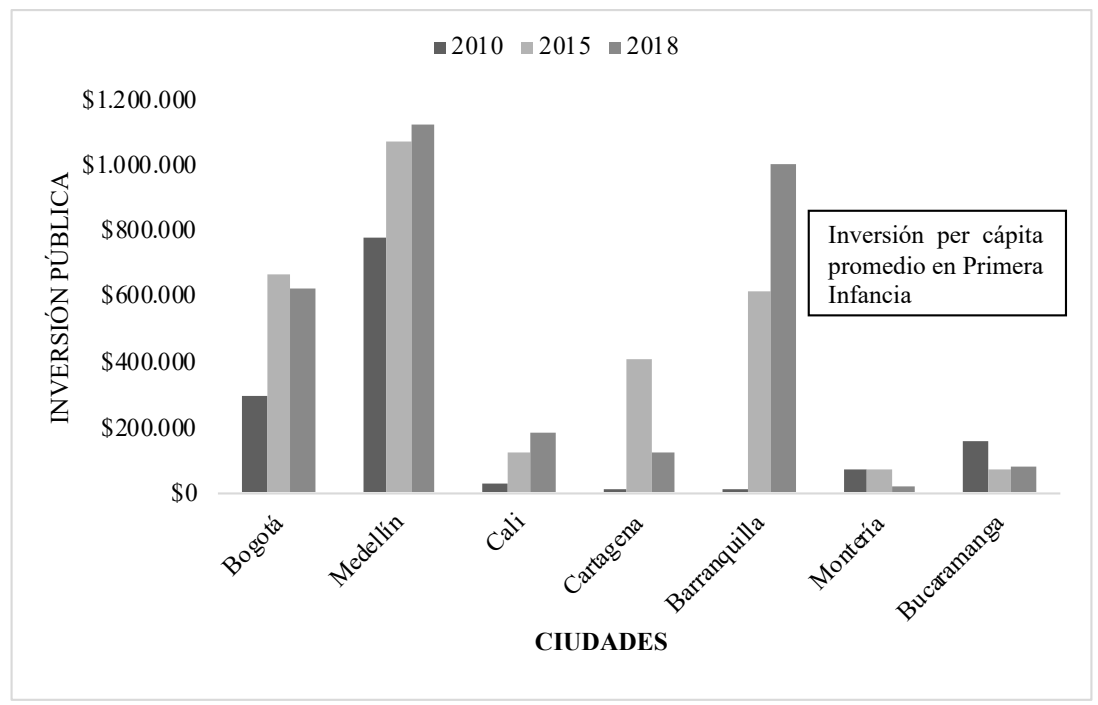

Nota. Basado en Formularios Únicos Territoriales (Ministerio de Hacienda, 2010, 2015, 2018).

La segunda tendencia corresponde a la concentración de la inversión distrital en el sector salud, decisión política que limita el cumplimiento de los compromisos adquiridos en materia de construcción de infraestructura para la atención institucional, tal como lo demanda la PPI (figura 4). Es así como, de las seis obras para la educación inicial propuestas, solo se han construido y entregado dos CDI, ambas obras con retrasos y sobrecostos.

\section{Figura 4}

Usos inversión en primera infancia en Cartagena y principales ciudades de Colombia (2010-2018)

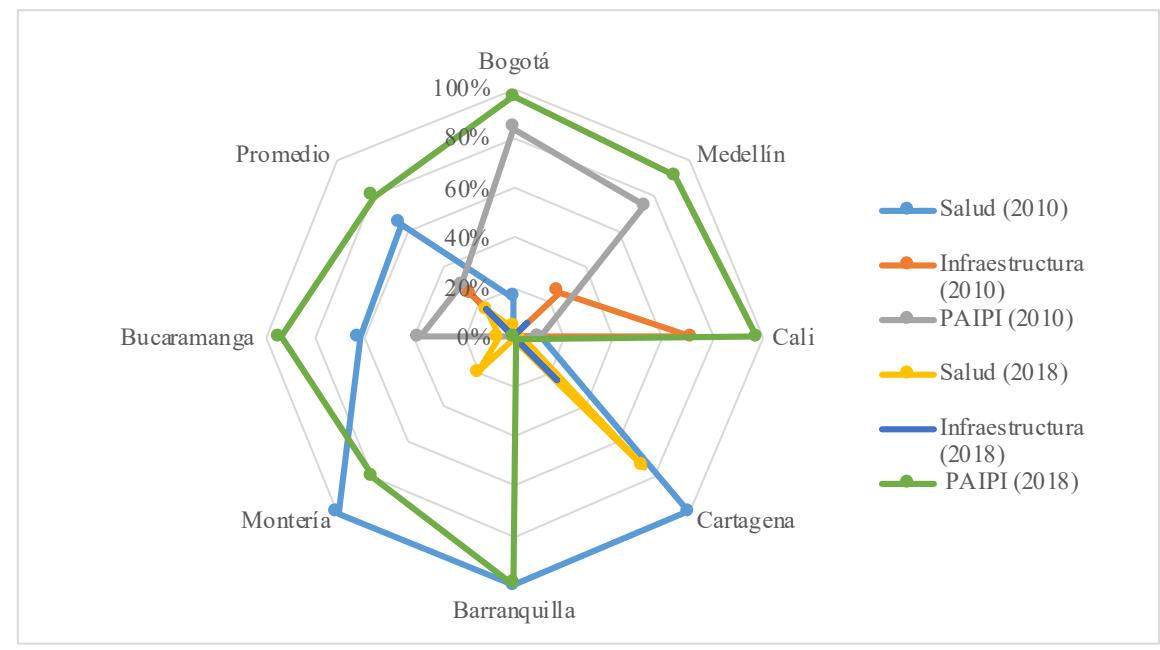

Nota. Basado en los Formularios Únicos Territoriales (Ministerio de Hacienda, 2010, 2018). 
La última tendencia se refiere a la dependencia de los recursos del Sistema general de participaciones para la inversión, que representaban en el año $2018 \mathrm{el} \mathrm{70} \mathrm{\%} \mathrm{del} \mathrm{total}$ de la inversión en primera infancia de la ciudad. Esta dependencia limita las posibilidades de saldar la deuda pendiente de la ciudad con la ampliación de cobertura con calidad para la atención integral a la primera infancia y la atención diferencial de comunidades étnicas.

Los aportes de los agentes pedagógicos de las EAS rurales e insulares develan en este contexto la subcategoría atención diferencial, la cual da cuenta del reconocimiento en la PPI de los niños y las niñas como «ciudadanos sujetos de derechos, seres sociales y singulares, e inmensamente diversos» (ICBF, 2017). La defensa de la diversidad y no discriminación étnica en la política atiende a marcos jurídicos internacionales y nacionales, como la Declaración y el Programa de Acción de Durban 2001, el Convenio 169 de la OIT, Ley 70 de 1993, Decreto 1745 de 1995, entre otros, que justifican la implementación de la modalidad propia e intercultural del ICBF.

Esta modalidad de atención busca promover la garantía de derechos, la participación y el desarrollo integral de la primera infancia étnica, respondiendo a las características propias de los territorios, a sus pautas de crianza, al fortalecimiento y la pervivencia cultural (ICBF, 2017). Se implementa en las Unidades Comunitarias de Atención concertadas con las comunidades, bajo los seis componentes de la atención definidos en la PPI (ICBF, 2014).

En Cartagena, las comunidades negras insulares, reconocidas por la Ley 70 de 1993 , se caracterizan por concentrar los más altos índices de pobreza y miseria, $96.6 \%$ y 55.1\% respectivamente (Cartagena Cómo Vamos, 2018); estas dos condiciones les ubican como zonas de alta prioridad para la implementación de los programas de atención de la PPI y de inversiones estatales que garanticen el acceso igualitario a los derechos. No obstante, la política pública de primera infancia no responde a esta condición de vulnerabilidad. Según los datos de atención recabados, el 100 \% de estas comunidades carecen de centros institucionales propios para la atención y educación inicial de la primera infancia (mapa 1); además, en el año 2019, solo el 1\% de estas comunidades contó con la operación del Programa de Atención Intercultural en su territorio.

A esto se le suma, según los agentes insulares, la desatención institucional específica en la garantía de derechos en las áreas de protección, desarrollo integral y educación formal con calidad y pertinencia. 
Acá es muy difícil que venga la Registraduría, el Dadis y otras entidades para hacer actividades constantes con los niños. Además, con el ICBF pasa algo, que los recursos que brinda para la compra de materiales para desarrollar las actividades pedagógicas con los niños son escasos, nos toca a nosotras sacar plata de nuestro bolsillo para comprar una colchoneta, platos, vasos, arreglar la casa, y todo eso lo evalúa el ICBF. (Madre comunitaria-15, 2019)

\section{Mapa 1}

\section{Centros de Atención y Cuidado en Cartagena (2019)}

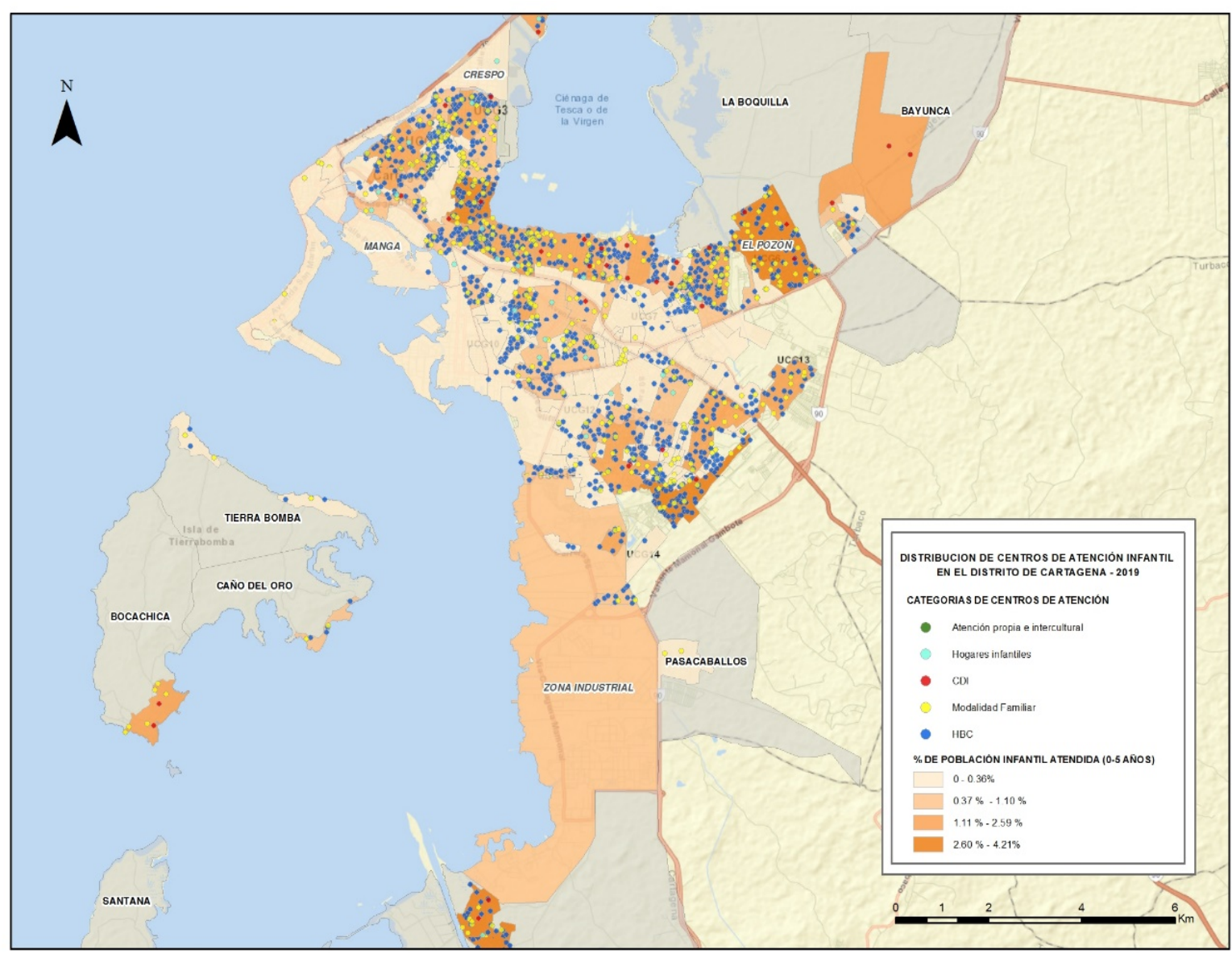

Nota. Basado en datos administrativos (ICBF, 2019).

Estos hechos constituyen condiciones desiguales en el acceso de los niños y las niñas de las comunidades negras a los derechos reconocidos en tratados internacionales y políticas nacionales. Estas asimetrías y limitaciones de tipo administrativo, gestión intersectorial, inversión y políticas públicas locales impiden en Cartagena el cumplimiento del principio de la PPI dirigido a la promoción y materialización de la atención integral, inclusiva e intercultural. 


\section{Discusión}

Este trabajo describe y analiza la implementación de la política pública de primera infancia en Cartagena de Indias, con el fin de identificar las principales barreras y oportunidades para la garantía de los derechos de los niños y niñas menores de cinco años de la ciudad, aportando así insumos que permitan el logro de su objetivo superior: la protección integral de la primera infancia.

En primer lugar, es necesario afirmar que la ratificación de la Convención sobre los Derechos del Niño y su implementación a partir de la formulación de políticas públicas para la atención a la infancia significa un avance importante en el reconocimiento de sus derechos. Asimismo, como lo afirma Galvis (2009), «abre el mundo de la niñez como instancia de la vida que tiene sus características propias y que deben ser reconocidas por la familia, la sociedad y los Estados» (p. 593). En este sentido, la política de atención integral a la primera infancia amplía las oportunidades de acceso a la protección y desarrollo integral de los niños y las niñas menores de 5 años en el país, pues obliga a cada una de las entidades del Sistema nacional de bienestar familiar a gestar los procesos institucionales necesarios para concretar el goce efectivo de estos derechos, especialmente de los niños y niñas más vulnerables.

Este estudio no es ajeno al contexto de la ciudad y sus dinámicas socioeconómicas; en consecuencia, el análisis de la política pública se enmarca en el reconocimiento de: primero, la desigualdad social que ubica a Cartagena como la ciudad con el mayor nivel de pobreza entre las principales ciudades de Colombia (Cartagena Cómo Vamos, 2018); segundo, de los impactos diferenciales provocados por las altas tasas de mortalidad causadas por enfermedades prevenibles y por la desnutrición; y, tercero, la exclusión social asociada a la condición étnica-racial de los niños y las niñas de la ciudad.

Los resultados evidenciaron que, a pesar de los avances normativos, en la ciudad persisten barreras para la implementación de la política pública y, por ende, el desarrollo integral y la garantía de los derechos de los niños y las niñas menores de cinco años, entre ellas se encuentran: 1) que existe una limitada infraestructura física para la oferta de la atención institucional en los CDI y Hogares Infantiles (especialmente en las zonas insulares en las que la población afrocolombiana es superior al 80\%); 2) no se están desarrollando los Programas de Atención Intercultural en los territorios étnicos de la ciudad; 3 ) persisten asimetrías en el acceso de los niños y las niñas a los programas de atención 
integral; y 4) existen barreras para consolidar un trabajo intersectorial que permita aunar esfuerzos para el cumplimiento de los derechos en salud, nutrición, cuidado y educación inicial a todos los niños y las niñas de la ciudad.

Según Bernal (2014), si bien la política está concebida para ser atendida territorialmente a partir de las Mesas Intersectoriales y los Consejos de Política Social, este nivel de coordinación y articulación entre sectores se desarrolla más armónicamente a nivel nacional. En Cartagena esta articulación es débil y se profundiza, entre otras razones, por la inestabilidad política e institucional que ha padecido la ciudad en los últimos ocho años, generada por los cambios de gobierno. Adicionalmente, algunos entrevistados afirman que la falta de voluntad política y el desconocimiento de algunas entidades de sus obligaciones dificultan la coordinación de acciones para la atención de los niños y las niñas en el marco de la política pública.

Una de las consecuencias de la débil articulación interinstitucional son las dificultades que se han presentado en la ciudad para asegurar el tránsito armonioso de los niños y las niñas a la educación formal. En este sentido, Alvarado y Suárez (2010) afirman que, en el país, el tema de las transiciones apenas empieza a estar en las agendas de las instancias centrales de formulación y aún no llega a las entidades territoriales como un aspecto determinante en los procesos educativos de los niños y niñas. Por tanto, el reto es lograr definir las estrategias a nivel pedagógico y de articulación intersectorial, así como coordinar los programas y las redes interinstitucionales alrededor de las prácticas y aplicación de dichas estrategias.

Por otra parte, garantizar la cobertura y transitar hacia la atención integral es uno de los grandes retos de la implementación de la política pública. En Cartagena se encontró una escasa dotación de infraestructura propia para el cuidado y protección infantil que limita la atención en iguales condiciones a los niños y las niñas menores de cinco años y reduce las posibilidades de tránsito a modalidades institucionales como lo pretende la PPI.

La deficiente y escasa infraestructura en Cartagena es consecuencia de los bajos niveles de inversión pública y la alta dependencia de recursos del Sistema general de participaciones en el presupuesto de primera infancia de la ciudad, lo que se refleja en el incumplimiento de las metas propuestas en los planes de desarrollo para garantizar la atención integral en las distintas áreas del desarrollo infantil. 
Otra de las debilidades identificadas en el estudio - y que coincide con lo que sucede en el resto del país- es la existencia de brechas educativas que se amplían entre zonas urbanas y rurales. Según Andrew et al. (2016), solo el $38 \%$ de los centros en Colombia cuentan con el equipo multidisciplinario necesario para atender las principales tareas de nutrición, revisión de menús, supervisión, monitoreo de casos especiales, seguimiento, entre otras actividades necesarias para la atención integral y de calidad de los menores de cinco años. La situación de las zonas insulares de Cartagena es mucho peor: según la información recopilada, los niños y las niñas que habitan en estas zonas carecen de centros institucionales propios para la atención y educación inicial de la primera infancia, y no cuentan con acompañamiento institucional ni con los profesionales necesarios para la garantía de derechos en las áreas de protección, desarrollo integral y educación formal con calidad. Esto, pese a la ordenanza de la PPI a los entes territoriales de atender de forma prioritaria a estas poblaciones, lo que expresa desigualdades en la protección de derechos.

Los resultados obtenidos permiten comprobar la hipótesis del estudio y afirmar que, entre los años 2015 y 2019, la oferta de servicios para el cuidado y atención a la primera infancia en Cartagena es desarticulada y desigual, y los recursos para su funcionamiento a nivel territorial no están garantizados. Por ende, su impacto en la inclusión social de los más pobres y la reducción de inequidades es marginal.

No obstante lo anterior, la vigencia de la PPI ha generado oportunidades y permitido: primero, un posicionamiento de la atención y garantía de los derechos de la primera infancia en la agenda política de la ciudad, que se refleja en la inclusión en los planes de desarrollo de estrategias, programas y presupuestos específicos para la atención de la primera infancia; segundo, pensar la primera infancia desde los escenarios de participación e incidencia ciudadana como la Mesa de Infancia y el Consejo de Política Social; y, tercero, concretar acciones de coordinación entre los diferentes sectores y actores, para sumar esfuerzos y cumplir con los compromisos adquiridos.

Si bien este estudio ayuda a comprender las causas políticas e institucionales que limitan el acceso a la atención con calidad, igualdad y pertinencia de los niños y las niñas menores de 5 años en Cartagena, es necesario ampliar el análisis a los determinantes sociales de la atención a la primera infancia en la ciudad, así como incluir a los niños y niñas que, a pesar de tener el derecho, no son atendidos por la política pública y las razones por las cuales esto sucede; lo anterior, de tal forma que se puedan generar los cambios necesarios para garantizar el desarrollo integral de los niños y niñas menores de cinco 
años más vulnerables de Cartagena, avanzando hacia el objetivo de reducir de la inequidad social en la ciudad. En palabras de Mieles y Acosta (2012):

El desafío no es solamente de carácter académico e investigativo sino de la necesaria revisión crítica y lucha por la transformación de las condiciones estructurales de desarrollo de los países que impiden el disfrute de una buena calidad de vida a la niñez, así como de la consecuente formulación de políticas públicas en favor de la infancia. (p. 214)

\section{Agradecimientos}

Las autoras agradecen, de manera especial, a las madres comunitarias, a los agentes docentes y a los coordinadores de los Centros de Desarrollo Infantil por la ayuda recibida en el desarrollo de la investigación. Igualmente, reconocen el apoyo invaluable de Maristella Madero (Fundación Grupo Social), Ricardo Senior (Caja de Compensación Familiar FenalcoAndi), Liliana Giraldo (Conviventia) y Berenice Camacho (Fundación Hogar Juvenil), en la discusión sobre la política pública de primera infancia en Cartagena.

\section{Referencias}

Alvarado, S. V., \& Suárez, M. (2010). Transiciones educativas en primera infancia, una mirada desde las políticas públicas en Colombia: avances y desafíos. Centro de Estudios Avanzados en Niñez y Juventud, Alianza Cinde-Universidad de Manizales.

Andrew, A., Attanasio, O., Bernal, R., Cardona, S., Krutikova, S., \& Rubio, M (2016).

Preschool quality and child development. Institute for Fiscal Studies, Economic \& Social Research Council. https://doi.org/10.1920/wp.ifs.2019.1923

Arrieta-Flórez, R., \& Caro-Gómez, E. E. (2010). Determinantes sociales y disparidades de la morbilidad por enfermedades respiratorias agudas (ERA) en niñas y niños de uno a cuatro años en Colombia. Gerencia y Políticas de Salud, 9(18), 137-156.

Ayala, J. \& Meisel, A. (2016). Cartagena libre de pobreza extrema en el 2033. Banco de la República. Baker, J., Gruber, J, \& Milligan, K. (2019). The long-run impacts of a universal child care program. American Economic Journal: Economic Policy, 11(3), 1-26. https://doi.org/gf5mgq

Bernal, R. (2014). Diagnóstico y recomendaciones para la atención de calidad a la primera infancia. Fedesarrollo. https://bit.ly/3lA4leN 
Bernal, R., \& Camacho, A. (2010). La importancia de los programas para la primera infancia en Colombia. Centro de Estudios sobre Desarrollo Económico.

Bernal, R., \& Camacho, A. (2012). La política de primera infancia en el contexto de la equidad $y$ movilidad social en Colombia. Universidad de Los Andes. https://bit.ly/3jsr8qk

Bernal, R., Fernández, C., Gaviria, A., Flórez, C., Ocampo, P., Sánchez, F., \& B. Samper, B. (2009). Evaluación de impacto del programa hogares comunitarios del Bienestar Familiar.

Bernal, R., \& Ramírez, S. (2019). Improving the quality of early childhood care at scale: The effects of «From Zero to Forever». World Development, 118, 91-105. https:// doi.org/10.1016/j.worlddev.2019.02.012

Black, M., Walker, S., Fernald, L., Andersen, C., DiGirolamo, A., \& Chunling, L. (2017). Early childhood development coming of age: Science through the life couse. Lancet, 389(10064), 77-9o. https://doi.org/10.1016/so140-6736(16)31389-7

Cartagena Cómo Vamos. (2018). ¿Para dónde vamos? Informe de calidad. Retos del próximo alcalde de Cartagena. https://bit.ly/2 $\mathrm{Voz}_{\mathrm{T}} \mathrm{l}$,

Chimá, W. (2020a, 19 de febrero). No se han cerrado los CDI, la contratación se retrasó. El Universal. https://bit.ly/3CovozO

Chimá, W. (2020b, 26 de febrero). «¿Qué tuvo en cuenta el ICBF para la contratación?»: preguntan operadores. El Universal. https://bit.ly/37toiM5

Comisión Económica para América Latina y el Caribe, \& Fondo de las Naciones Unidas para la Infancia (2018). América Latina y el Caribe a 30 años de la aprobación de la Convención sobre los Derechos del Niño. https://bit.ly/3rWFvHb

Comisión Intersectorial para la Atención Integral a la Primera Infancia. (2013). Estrategia de atención integral a la primera infancia: fundamentos políticos, técnicos y de gestión. Imprenta Nacional. https://bit.ly/3CqMddm

Comisión Económica para América Latina y el Caribe \& Fondo de las Naciones Unidas para la Infancia. (2018). América Latina y el Caribe a 30 años de la aprobación de la Convención sobre los Derechos del Niño. Autores.

Congreso de la República de Colombia. (2006). Ley 1098. Mediante la cual se promulga el Código de la Infancia y la Adolescencia.

Congreso de la República de Colombia. (2016). Ley 1804. Por la cual se establece la política de Estado para el Desarrollo Integral de la Primera Infancia de Cero a Siempre y se dictan otras disposiciones.

Fondo de las Naciones Unidas para la Infancia. (2006). Convención sobre los Derechos del Niño. https://bit.ly/3xvub63 
Galvis, L. (2009). La Convención de los Derechos del Niño veinte años después. Revista Latinoamericana de Ciencias Sociales, Niñez y Juventud, 7(2), 587-619.

Gómez, Z. (2007). El trabajo infantil en clave colonial: consideraciones histórico-antropológicas. Nómadas, (26), 80-90.

Hernández-Sampieri, R., \& Batista, L. (2014). Metodología de la investigación. McGraw- Hill. Hernández-Sampieri, R., \& Mendoza, C. (2018). Metodología de la investigación: las rutas cuantitativa, cualitativa y mixta. McGraw-Hill Education.

Instituto Colombiano de Bienestar Familiar. (2014). Manual técnico operativo servicio de educación inicial en el marco de la atención integral para la primera infancia-modalidad propia e intercultural para comunidades étnicas y rurales. Autor.

Instituto Colombiano de Bienestar Familiar. (2017). Manual técnico operativo. Servicio de educación inicial en el marco de la atención integral para la primera infancia: modalidad propia e intercultural para comunidades étnicas y rurales. https://bit.ly/3fBeTGQ

Leseman, P., \& Slot, P. (2020). Universal versus targeted approaches to prevent Early education gaps. The Netherlands as case in point. Erziehungswiss, (23), 485-507. https://doi.org/10.1007/s11618-020-00948-8

Mieles, M. D., \& Acosta, A. (2012). Calidad de vida y derechos de la infancia: un desafío presente. Revista Latinoamericana de Ciencias Sociales, Niñez y Juventud, 10(1), 205-217.

Ministerio de Hacienda (2020, 20 de enero). Formularios Únicos Territoriales. [Fichero de datos]. https://www.chip.gov.co/schip_rt/index.jsf

Nores, M., \& Barnett, S. (2010). Benefits of Early childhood inventions across the world: (Under) investing in the very young. Economic of Education Rewiew, 29(2), 271-282. https://doi.org/10.1016/j.econedurev.2009.09.001

Organización de las Naciones Unidas. (2005). Observación General № 7 del Comité de los Derechos del Niño: realización de los derechos del niño en la primera infancia. https:// bit.ly/obs7unicef

Rodríguez, D. R., \& Benavides, J. A. (2016). Salud y ruralidad en Colombia: análisis desde los determinantes sociales de la salud. Revista Facultad Nacional de Salud Pública, 34(3), 359-371. https://doi.org/10.17533/udea.rfnsp.v34nza10

Rodríguez, M. (2014). La educación inicial y la educación preescolar: perspectivas de desarrollo en Colombia y su importancia en la configuración del mundo de los niños. Cultura, Educación y Sociedad, 5(2), 97-111.

Sheldom, S. (2019). Inclusive education: A prerequisite for equity and social justice. Asia Pacific Education Review. 